\title{
MOSCO CONVERGENCE AND REFLEXIVITY
}

\author{
GERALD BEER AND JONATHAN M. BORWEIN
}

(Communicated by William J. Davis)

\begin{abstract}
In this note we aim to show conclusively that Mosco convergence of convex sets and functions and the associated Mosco topology $\tau_{M}$ are useful notions only in the reflexive setting. Specifically, we prove that each of the following conditions is necessary and sufficient for a Banach space $X$ to be reflexive: (1) whenever $A, A_{1}, A_{2}, A_{3}, \ldots$ are nonempty closed convex subsets of $X$ with $A=\tau_{M}-\lim A_{n}$, then $A^{\circ}=\tau_{M}-\lim A_{n}^{\circ} ;(2) \tau_{M}$ is a Hausdorff topology on the nonempty closed convex subsets of $X ;(3)$ the arg min multifunction $f \rightrightarrows\left\{x \in X: f(x)=\inf _{X} f\right\}$ on the proper lower semicontinuous convex functions on $X$, equipped with $\tau_{M}$, has closed graph.
\end{abstract}

\section{INTRODUCTION}

Let $\mathscr{C}(X)$ be the nonempty closed convex subsets of a Banach space $X$. A well-studied notion of convergence of sequences of convex sets in reflexive spaces, from the point of view of both optimization and Banach space geometry [At, BP, Be1, Be2, BF, LP, Mc, Mo1, Mo2, SW, So, Ts], is that of Mosco convergence. Specifically, a sequence $\left\langle A_{n}\right\rangle$ in $\mathscr{C}(X)$ is declared Mosco convergent to $A \in \mathscr{C}(X)$ provided both of the following conditions hold:

(Mi) for each $a \in A$ there exists a sequence $\left\langle a_{n}\right\rangle$ convergent in norm to $a$ such that for each $n, a_{n} \in A_{n}$;

(Mii) whenever $n(1)<n(2)<\cdots$ and $a_{k} \in A_{n(k)}$ for each $k \in Z^{+}$, then the weak $\left(\sigma\left(X, X^{*}\right)\right)$ convergence of $\left\langle a_{k}\right\rangle$ to $x \in X$ implies $x \in A$.

Perhaps the most important feature of Mosco convergence in reflexive spaces is its stability with respect to duality, as expressed by the (sequential) continuity of the polar map $A \rightarrow A^{\circ}$, or by the (sequential) continuity of the conjugate map $f \rightarrow f^{*}$ defined on $\Gamma(X)$, the proper lower semicontinuous convex functions $X$, where functions are identified with their epigraphs. These results are due to Mosco in [Mo2], although they were first obtained in finite

Received by the editors May 12, 1989 and, in revised form, August, 20, 1989.

1980 Mathematics Subject Classification (1985 Revision). Primary 46B20; Secondary 54B20, $54 \mathrm{C} 60$.

Key words and phrases. Mosco convergence, polar convex set, conjugate convex function, arg min multifunction, reflexivity.

Research of the second author was partially supported by NSERC. 
dimensions by Wijsman [Wi]. In [Bel] a simple Vietoris-type topology $\tau_{M}$ compatible with Mosco convergence of sequences in any Banach space was introduced, and in [Be2], Mosco's sequential continuity results were extended to topological continuity theorems with respect to $\left\langle\mathscr{C}(X), \tau_{M}\right\rangle$, and $\left\langle\Gamma(X), \tau_{M}\right\rangle$, respectively (for reflexive $X, \tau_{M}$ is first countable if and only if $X$ is separable, so that sequences do not otherwise determine the topology).

The above results-and all other positive results of any depth regarding Mosco convergence-invariably assume reflexivity of the underlying space. We show in this paper that reflexivity assumptions are indeed required. As a main result, we show that even sequential continuity of the polar map must fail without reflexivity.

\section{NOTATION AND SOME BACKGROUND MATERIAL}

Unless otherwise stated, $X$ will denote a Banach space with origin $\theta$ and closed unit ball $U$. The continuous dual of $X$ will be denoted by $X^{*}$. For each $A \in \mathscr{C}(X)$ its polar $A^{\circ}$ is the following weak star $\left(\sigma\left(X^{*}, X\right)\right)$ closed subset of $X^{*}: A^{\circ} \equiv\left\{y \in X^{*}: y(a) \leq 1\right.$ for each $\left.a \in A\right\}$. The epigraph of an extended real valued function $f$ on $X$, as a subset of $X \times R$, is defined by

$$
\text { epi } f \equiv\{(x, \alpha): x \in X, \alpha \in R \text {, and } \alpha \geq f(x)\} \text {. }
$$

The function is called proper if epi $f$ is nonempty and contains no vertical line. We denote the sublevel set $\{x \in X: f(x) \leq \alpha\}$ at height $\alpha$ of $f$ by $\operatorname{lev}(f ; \alpha)$. For each $f \in \Gamma(X)$, its conjugate $f^{*} \in \Gamma\left(X^{*}\right)$ is defined at each $y \in X^{*}$ by $f^{*}(y)=\sup \{y(x)-f(x): x \in X\}$. The condition $(y, \alpha) \in$ epi $f^{*}$ means that $f$ majorizes $x \rightarrow y(x)-\alpha$. By $\arg \min f$, we mean the (possibly empty) set of minimizers of $f: \arg \min f \equiv\left\{x \in X: f(x)=\inf _{X} f\right\}$.

To describe the topology of Mosco convergence $\tau_{M}$ on $\mathscr{C}(X)$, we need some standard notation from the theory of hyperspaces (topologies on sets of subsets). If $E \subset X$, we define subsets $E^{-}$and $E^{+}$of $\mathscr{C}(X)$ as follows:

$$
E^{-} \equiv\{A \in \mathscr{C}(X): A \cap E \neq \varnothing\} \quad E^{+} \equiv\{A \in \mathscr{C}(X): A \subset E\} .
$$

The Mosco topology $\tau_{M}$ [Be1] on $\mathscr{C}(X)$ has as subbase all sets of the form $V^{-}$where $V$ is strongly open, and $\left(K^{C}\right)^{+}$where $K$ is weakly compact. Since $\left(\left(K_{1} \cup K_{2}\right)^{C}\right)^{+}=\left(K_{1}^{C}\right)^{+} \cap\left(K_{2}^{C}\right)^{+}$, all sets of the following form determine a base for the topology:

$$
\left[V_{1}, V_{2}, \ldots, V_{n} ; K\right] \equiv\left(\bigcap_{i=1}^{n} V_{i}^{-}\right) \cap\left(K^{C}\right)^{+},
$$

where $V_{1}, V_{2}, \ldots, V_{n}$ are strongly open, and $K$ is weakly compact. Since elements of $\Gamma(X)$ may be viewed as nonempty closed convex subsets of $X \times R$, we may consider the function space as a topological subspace of $\left\langle\mathscr{C}(X \times R), \tau_{M}\right\rangle$. It is in this sense that $\left\langle\Gamma(X), \tau_{M}\right\rangle$ is to be understood. Of course, Mosco convergence of sequences of convex functions may also be defined directly (see Lemma 1.10 of [Mol] or [At, p. 297]). 


\section{Polarity is not continuous unless $X$ is Reflexive}

As noted in the introduction, if $X$ is reflexive, then the polar map from $\left\langle\mathscr{C}(X), \tau_{M}\right\rangle$ to $\left\langle\mathscr{C}\left(X^{*}\right), \tau_{M}\right\rangle$ is continuous. Here, we show that the converse holds. The main idea is this: If $X$ is not reflexive, there is a norm one element $z$ of $X^{*}$ that is not norm attaining on the unit ball. From this, we construct a sequence $\left\langle C_{n}\right\rangle$ in $\mathscr{C}(X)$ Mosco convergent to $C=\{\theta\}$ such that the points of $X^{*}$ that are strong limits of sequences of points chosen from $\left\langle C_{n}^{\circ}\right\rangle$ are constrained to lie in a closed halfspace determined by a Hahn-Banach functional for $z$. Thus, $\left\langle C_{n}^{\circ}\right\rangle$ cannot converge to $C^{\circ}=X^{*}$ in the sense of Mosco.

A preparatory lemma will be helpful.

Lemma 3.1. Let $\left\langle A_{n}\right\rangle$ be a decreasing sequence of nonempty closed and bounded convex subsets of $X$ with empty intersection. If for each $n, C_{n}=\operatorname{co}\left(\{\theta\} \cup A_{n}\right)$, then $C_{n} \in \mathscr{C}(X)$ and $\bigcap_{n \geq 1} C_{n}=\{\theta\}$.

Proof. Clearly, $\theta \in \bigcap_{n \geq 1} C_{n}$. Suppose $c \in \bigcap_{n \geq 1} C_{n}$ and $c \neq \theta$. For each $n, c=\lambda_{n} a_{n}$ where $\lambda_{n} \in[0,1]$ and $a_{n} \in A_{n}$. By passing to a subsequence we may assume that $\left\langle\lambda_{n}\right\rangle$ converges to $\lambda$. Clearly, $\lambda \neq 0$, or else $c$ would be the origin, because $\left\langle a_{n}\right\rangle$ is uniformly bounded. But then $c / \lambda=\lim _{n \rightarrow \infty} c / \lambda_{n}=$ $\lim _{n \rightarrow \infty} a_{n}$, yielding $\bigcap_{n \geq 1} A_{n} \neq \varnothing$. Thus, $\bigcap_{n \geq 1} C_{n}=\{\theta\}$.

Theorem 3.2. Suppose $X$ is a Banach space such that whenever $C, C_{1}, C_{2}, \ldots$ are closed and bounded convex sets with $C=\tau_{M}-\lim C_{n}$, then $C^{\circ}=\tau_{M}-$ $\lim C_{n}^{\circ}$. Then $X$ is reflexive.

Proof. Suppose $X$ is not reflexive. By James's theorem, there is a norm one functional $z \in X^{*}$ that does not attain its norm on the closed unit ball (see [Da, p. 63] or [Ja]). Consider for $n \in Z^{+}$the closed bounded convex set

$$
A_{n} \equiv\left\{x \in X: z(x) \geq 1-2^{-n} \text { and }\|x\| \leq 1\right\},
$$

and let

$$
C_{n} \equiv \operatorname{co}\left(\{\theta\} \cup A_{n}\right)=\left\{\alpha x: x \in A_{n} \text { and } 0 \leq \alpha \leq 1\right\} .
$$

We will show: (a) $\tau_{M}-\lim C_{n}=C=\{\theta\}$, but that (b) $\tau_{M}-\lim C_{n}^{\circ} \neq C^{\circ}=$ $X^{*}$ (we make no claim that $\tau_{M}-\lim C_{n}^{\circ}$ exists).

(a) Since $\theta \in C_{n}$ for each $n$, (Mi) holds. To show (Mii) holds, suppose that $c_{k} \in C_{n(k)}$ for $k=1,2,3, \ldots$, and $\left\langle c_{k}\right\rangle$ converges weakly to $x$. Since $\left\langle C_{n}\right\rangle$ is a decreasing sequence of weakly closed sets, we get $x \in C_{n}$ for each $n$. Thus, by Lemma 3.1, $x=\theta \in C$ as required.

(b) Since $z$ has unit norm in $X^{*}$, there is an element $F$ of $X^{* *}$ such that $F(z)=\|F\|=1$. Suppose for each $n, y_{n} \in C_{n}^{\circ}$ and $\left\langle y_{n}\right\rangle$ converges to $y_{0}$ in norm. We will show that $F\left(y_{0}\right) \leq 1$, so that condition (Mi) fails for any point of $C^{\circ}=X^{*}$ that fails to lie in the closed half space $\left\{y \in X^{*}: F(y) \leq 1\right\}$.

Consider this weak neighborhood of $F$ defined by

$$
W_{n} \equiv\left\{G \in X^{* *}:|F(z)-G(z)|<2^{-n} \text { and }\left|F\left(y_{0}\right)-G\left(y_{0}\right)\right|<2^{-n}\right\} .
$$


By Goldstein's density theorem [Da, p. 47], for each $n$, there is a point $x_{n} \in X$ (appropriately identified as a subspace of $X^{* *}$ ) with $x_{n} \in W_{n}$ and with $\left\|x_{n}\right\| \leq$ 1. In particular, $z\left(x_{n}\right) \geq 1-2^{-n}$ so that $x_{n} \in C_{n}$. Since $y_{n} \in C_{n}^{\circ}$, we have $y_{n}\left(x_{n}\right) \leq 1$. From the definition of $W$ again, $\left|F\left(y_{0}\right)-y_{0}\left(x_{n}\right)\right|<2^{-n}$. We obtain

$$
\begin{aligned}
F\left(y_{0}\right) \leq y_{0}\left(x_{n}\right)+2^{-n} & \leq y_{n}\left(x_{n}\right)+\left\|y_{n}-y_{0}\right\| \cdot\left\|x_{n}\right\|+2^{-n} \\
& \leq 1+\left\|y_{n}-y_{0}\right\|+2^{-n} .
\end{aligned}
$$

As $\left\langle y_{n}\right\rangle$ is convergent in norm to $y_{0}$, we get $F\left(y_{0}\right) \leq 1$, as desired.

We note that in [Be4], an example is given showing that $\tau_{M}$-continuity of $C \rightarrow C^{\circ}$ fails for sequences of hyperplanes in the nonreflexive space $l_{1}$. The construction is completely unrelated to the one presented above. Indeed there are nonreflexive spaces, such as $c_{0}$, for which $\tau_{M}$-continuity of $C \rightarrow C^{\circ}$ holds for sequences of hyperplanes.

From Theorem 3.2, it easily follows that the conjugate operator fails to be sequentially $\tau_{M}$-continuous in nonreflexive spaces.

Theorem 3.3. Suppose $X$ is a Banach space such that whenever $f, f_{1}, f_{2}, \ldots$ are proper lower semicontinuous convex functions on $X$ with $f=\tau_{M}-\lim f_{n}$, then $f^{*}=\tau_{M}-\lim f_{n}^{*}$. Then $X$ is reflexive.

Proof. Let $\delta(\cdot, A)$ be the indicator function of a closed convex set $A$. As is well known, $\delta^{*}(\cdot, A)$ is the support function for $A, A^{\circ}=\operatorname{lev}\left(\delta^{*}(\cdot, A) ; 1\right)$ [Ho, §14]. Suppose $X$ is not reflexive, and the conjugate map is sequentially continuous. Let $C$ and $\left\langle C_{n}\right\rangle$ be as in the proof of Theorem 3.2. Then $\delta(\cdot, C)=\tau_{M}-\lim \delta\left(\cdot, C_{n}\right)$ so that $\delta^{*}(\cdot, C)=\tau_{M}-\lim \delta^{*}\left(\cdot, C_{n}\right)$. But since Mosco convergence of convex functions ensures Mosco convergence of sublevel sets at a fixed height exceeding the infimum of the limit [Be2, Lemma 3.2], we recover $C^{\circ}=\tau_{M}-\lim C_{n}^{\circ}$. This is a contradiction to Theorem 3.2.

\section{4. $\tau_{M}$ IS NOT HAUSDORFF UNLESS $X$ IS REFLEXIVE}

If $X$ is a reflexive Banach space, it is immediate that $\left\langle\mathscr{C}(X), \tau_{M}\right\rangle$ is Hausdorff. To see this, if $A \in \mathscr{C}(X)$ and $C \in \mathscr{C}(X)$, and $A \cap C^{C} \neq \varnothing$, then there exists $a \in A$ and $\varepsilon>0$ such that $(a+\varepsilon U) \cap C=\varnothing$. Clearly, $(a+\text { int } \varepsilon U)^{-}$and $\left((a+\varepsilon U)^{C}\right)^{+}$are disjoint $\tau_{M}$-open neighborhoods of $A$ and $C$, respectively. We show that when $X$ is nonreflexive, then each $\tau_{M}$-open set is actually dense in $\left\langle\mathscr{C}(X), \tau_{M}\right\rangle$. This falls out of a geometric lemma regarding the structure of weakly compact sets in a nonreflexive space.

Lemma 4.1. Let $X$ be a nonreflexive space. Let $K$ be a weakly compact subset of $X$ and let $V_{1}, V_{2}, \ldots, V_{n}$ be nonempty open subsets of $X$ (not necessarily distinct). Then there exist distinct points $x_{i} \in V_{i}$ such that $\operatorname{co}\left\{x_{1}, x_{2}, \ldots, x_{n}\right\} \cap$ $K=\varnothing$.

Proof. We rely on the fact that a weakly compact set in a nonreflexive space must have empty interior. Choose $\varepsilon>0$ and distinct $x_{i} \in V_{i}$ such $U_{i} \equiv$ $x_{i}+\varepsilon U \subset V_{i}$. For each $u \in U$, set $P(u) \equiv \operatorname{co}\left\{x_{1}+\varepsilon u, x_{2}+\varepsilon u, \ldots, x_{n}+\varepsilon u\right\}$, 
so that $P(u)=P(\theta)+\varepsilon u$. We claim that for some point $u \in U, P(u) \cap K=\varnothing$. Else, for each $u \in U,[P(\theta)+\varepsilon u] \cap K \neq \varnothing$, and $\varepsilon U \subset K-P(\theta)$. This last sum is weakly compact with nonempty norm interior. We conclude that, for some $u$ in $U, \operatorname{co}\left\{x_{1}+\varepsilon u, x_{2}+\varepsilon u, \ldots, x_{n}+\varepsilon u\right\} \cap K=\varnothing$.

Theorem 4.2. Let $X$ be a nonreflexive Banach space. Then neither $\left\langle\mathscr{C}(X), \tau_{M}\right\rangle$ nor $\left\langle\Gamma(X), \tau_{M}\right\rangle$ is Hausdorff.

Proof. Let $\left[V_{1}, V_{2}, \ldots, V_{m} ; K\right]$ and $\left[W_{1}, W_{2}, \ldots, W_{n} ; K^{\prime}\right]$ be basic $\tau_{M}$-open subsets of $\mathscr{C}(X)$. By Lemma 4.1 there exists $x_{i} \in V_{i}$ and $w_{i} \in W_{i}$ such that $\operatorname{co}\left\{x_{1}, x_{2}, \ldots, x_{m}, w_{1}, w_{2}, \ldots, w_{n}\right\} \cap\left(K \cup K^{\prime}\right)=\varnothing$. Clearly, the polytope $P \equiv \operatorname{co}\left\{x_{1}, x_{2}, \ldots, x_{m}, w_{1}, w_{2}, \ldots, w_{n}\right\}$ lies in the intersection of $\left[V_{1}, V_{2}\right.$, $\left.\ldots, V_{m} ; K\right]$ and $\left[W_{1}, W_{2}, \ldots, W_{n} ; K^{\prime}\right]$. Thus, no two $\tau_{M}$-open subsets of $\mathscr{C}(X)$ can be disjoint. From this, $\left\langle\Gamma(X), \tau_{M}\right\rangle$ must fail to be Hausdorff because $A \rightarrow \delta(\cdot, A)$ is an embedding of $\left\langle\mathscr{C}(X), \tau_{M}\right\rangle$ into $\left\langle\Gamma(X), \tau_{M}\right\rangle$.

\section{ON THE ARG MIN MULTIFUNCTION}

From the perspective of convex optimization, a minimal requirement that a topology $\tau$ on $\Gamma(X)$ should satisfy to be of any interest is the following: if $x_{\lambda}$ is a minimizer of $f_{\lambda}$ and $f_{0}=\tau-\lim f_{\lambda}$ and $\left\|x_{\lambda}-x_{0}\right\| \rightarrow 0$, then $x_{0}$ should be a minimizer of $f_{0}$. When $\tau=\tau_{M}$, we show that this condition is satisfied if and only if $X$ is reflexive.

The condition expressed in the preceding paragraph is conveniently described in the language of multifunctions. By a multifunction $\varphi: T \rightrightarrows X$ from a topological space $T$ to another topological space $X$, we mean a function that assigns to each $t \in T$ a possibly empty closed subset $\varphi(t)$ of $X$. The graph of $\varphi$ is this subset of $T \times X:\{(t, x): x \in \varphi(t)\}$. When $X$ is a Banach space, we say that $\varphi$ has strongly (resp. weakly) closed graph provided its graph is a closed subset of $T \times X$ when $X$ is equipped with the norm (resp. weak) topology. We have claimed that the arg min multifunction has strongly closed graph if and only if $X$ is reflexive. Remarkably, the multifunction fails to have weakly closed graph, even though this is true sequentially! Lemma 4.1 again plays a role in the analysis.

Theorem 5.1. Let $X$ be a Banach space. The following are equivalent:

(a) $X$ is reflexive;

(b) $\arg \min :\left\langle\Gamma(X), \tau_{M}\right\rangle \rightrightarrows X$ has strongly closed graph;

(c) whenever $f_{0}=\tau_{M}-\lim f_{\lambda}$ and $x_{0}=\sigma\left(X, X^{*}\right)-\lim x_{\lambda}$ where $x_{\lambda} \in$ $\arg \min f_{\lambda}$ and $\left\langle x_{\lambda}\right\rangle$ is eventually bounded, then $x_{0} \in \arg \min f_{0}$.

Proof. (a) $\Rightarrow$ (c). By passing to a subnet we may assume that for some $\rho>0$ and all $\lambda$, we have $\left\|x_{\lambda}\right\| \leq \rho$. Suppose to the contrary that $x_{0} \notin \arg \min f_{0}$. Choose $x_{1} \in X$ with $f_{0}\left(x_{1}\right)<f_{0}\left(x_{0}\right)$. Since $f_{0}$ is the supremum of the continuous affine functionals that it majorizes, there exists $(y, \beta) \in X^{*} \times R$ with $\beta>f_{0}^{*}(y)$ such that $y\left(x_{0}\right)-\beta>f_{0}\left(x_{1}\right)$. Choose $\beta_{0}$ strictly between $y\left(x_{0}\right)-\beta$ 
and $f_{0}\left(x_{1}\right)$. Then $H \equiv\left\{x \in X: y(x) \geq \beta_{0}+\beta\right\}$ is a weak neighborhood of $x_{0}$. By reflexivity, the following half-cylindrical slice of the graph of $x \rightarrow y(x)-\beta$ is a weakly compact subset of $X \times R$ :

$$
K \equiv\{(x, \alpha):\|x\| \leq \rho, x \in H \text { and } \alpha=y(x)-\beta\} .
$$

In fact, $K$ is the graph of a weakly continuous function restricted to a weakly compact set. The condition $\beta>f_{0}^{*}(y)$ guarantees that $f_{0} \in\left(K^{C}\right)^{+}$. Also, $f_{0} \in U^{-}$where $U \equiv X \times\left(-\infty, \beta_{0}\right)$ because $f_{0}\left(x_{1}\right)<\beta_{0}$. Take $\lambda_{0}$ in the underlying directed set for the net such that for all $\lambda \geq \lambda_{0}$, we have both $f_{\lambda} \in U^{-} \cap\left(K^{C}\right)^{+}$and $x_{\lambda} \in H$. Now epi $f_{\lambda} \cap K=\varnothing$ can occur only if epi $f_{\lambda}$ lies above $K$, for epigraphs recede in the vertical direction. As a result, for all $\lambda \geq \lambda_{0}$, we have

$$
f_{\lambda}\left(x_{\lambda}\right)>y\left(x_{\lambda}\right)-\beta \geq\left(\beta_{0}+\beta\right)-\beta=\beta_{0} .
$$

However, the condition $f_{\lambda} \in U^{-}$means that $\inf _{X} f_{\lambda}<\beta_{0}$. This contradicts our assumption that $x_{\lambda} \in \arg \min f_{\lambda}$.

(c) $\Rightarrow$ (b). This is obvious, for if a net $\left\langle x_{\lambda}\right\rangle$ in $X$ is convergent strongly, then it is eventually norm bounded.

(b) $\Rightarrow(a)$. Suppose $X$ is not reflexive. We show that

$$
\mathscr{A} \equiv\{(g, x): g \in \Gamma(X) \text { and }\{x\}=\arg \min g\}
$$

is dense in $\Gamma(X) \times X$, where $\Gamma(X)$ is equipped with the Mosco topology and $X$ is equipped with the norm topology. Let $(f, a)$ be an arbitrary point in the product, and let $\left[V_{1}, \ldots, V_{n} ; K\right]$ be an arbitrary $\tau_{M}$-neighborhood of $f$, and let $\varepsilon>0$. By Lemma 4.1, we can choose distinct points $x_{i} \in \pi_{X}\left(V_{i}\right)$ and $x_{0} \in \operatorname{int}(a+\varepsilon U)$ such that $\operatorname{co}\left\{x_{0}, x_{1}, \ldots, x_{n}\right\} \cap \pi_{X}(K)=\varnothing$. Let $P=$ $\operatorname{co}\left\{x_{0}, x_{1}, \ldots, x_{n}\right\}$. Since $P$ and $\pi_{X}(K)$ lie a positive distance apart and $\pi_{X}\left(V_{i}\right)$ is open for each $i$, there is no loss in generality in assuming that $\left\{x_{0}, x_{1}, \ldots, x_{n}\right\}$ is an affinely independent set. Thus, each point of $P$ may be uniquely represented as a convex combination of the vertices. For each $i \in$ $\{1,2, \ldots, n\}$, pick $\alpha_{i}$ with $\left(x_{i}, \alpha_{i}\right) \in V_{i}$, and take $\alpha_{0}<\min \left\{\alpha_{i}: 1 \leq i \leq n\right\}$. Then $g \in \Gamma(X)$ defined by

$$
g(x)= \begin{cases}\Sigma \lambda_{i} \alpha_{i} & \text { if } x=\Sigma \lambda_{i} x_{i} \text { with } \lambda_{i} \geq 0 \text { and } \Sigma \lambda_{i}=1, \\ \infty & \text { otherwise, }\end{cases}
$$

is well defined and, by construction, lies in $\left[V_{1}, \ldots, V_{n} ; K\right]$. Moreover, $\arg \min g=\left\{x_{0}\right\}$. Thus, $\left(g, x_{0}\right) \in \mathscr{A} \cap\left[V_{1}, \ldots, V_{n} ; K\right] \times(a+\varepsilon U)$, establishing density of $\mathscr{A}$ in $\Gamma(X) \times X$. As a result, the graph of the arg min multifunction is not strongly closed.

Without reflexivity, it is a routine exercise to check that arg min has weakly sequentially closed graph: If $x_{n}$ is a minimizer of $f_{n}$, and $x=\alpha\left(X, X^{*}\right)-$ $\lim x_{n}$ and $f=\tau_{M}-\lim f_{n}$, then $x$ must be a minimizer of $f$. Combining this with the equivalence of $(a)$ and $(b)$ in Theorem 5.1, we see that reflexivity of $X$ is necessary for first countability of $\left\langle\Gamma(X), \tau_{M}\right\rangle$. Taking note of the results 
of [Be1], we see that first countability of the function space for separable $X$ is equivalent to reflexivity of $X$.

Condition (c) in the statement Theorem 5.1 is formally weaker than weak closedness of the graph of arg min: $\left\langle\Gamma(X), \tau_{M}\right\rangle \rightrightarrows X$. We intend to show that weak closedness actually fails unless $X$ is finite dimensional. What goes wrong in infinite dimensions is that a weakly convergent net of minimizers of a Mosco convergent net of convex functions need not be eventually bounded. For this purpose, we present a characterization of $\tau_{M}$-convergence of nets, generalizing the sequential conditions (Mi) and (Mii), the essence of whose proof may be found in the proof of Theorem 5.2 of [Be3].

Lemma 5.2. Let $X$ be a reflexive Banach space, and let $\left\langle A_{\lambda}\right\rangle_{\lambda \in \Lambda}$ be a net in $\mathscr{C}(X)$. Then $A=\tau_{M}-\lim _{\lambda \in \Lambda} A_{\lambda}$ if and only if both of the following conditions are satisfied:

(M1) For each $a \in A$, each norm open neighborhood of a meets $\left\langle A_{\lambda}\right\rangle$ eventually; and

(M2) whenever $\Lambda^{\#}$ is a cofinal subset of $\Lambda$ and for each $\lambda \in \Lambda^{\#}$, we have $a_{\lambda} \in A_{\lambda}$, and $\left\langle a_{\lambda}\right\rangle_{\lambda \in \Lambda^{*}}$ is norm bounded, then $A$ contains each weak cluster point of $\left\langle a_{\lambda}\right\rangle_{\lambda \in \Lambda^{*}}$.

Proof. Evidently, (M1) is equivalent to the requirement that whenever $V$ is norm open and $A \in V^{-}$, then $\left\langle A_{\lambda}\right\rangle_{\lambda \in \Lambda}$ is in $V^{-}$eventually.

We show that (M2) holds if and only if for each weakly compact subset $K$ of $X$, whenever $\left\langle A_{\lambda}\right\rangle_{\lambda \in \Lambda}$ is in $K^{-}$frequently, then $A \in K^{-}=\left[\left(K^{C}\right)^{+}\right]^{C}$. Suppose first that (M2) holds, and for some cofinal subset $\Lambda^{\#}$ of $\Lambda$ and for each $\lambda \in \Lambda^{\#}$, we have $A_{\lambda} \cap K \neq \varnothing$. Pick $a_{\lambda}$ in the intersection. Since $K$ is weakly compact, $\left\langle a_{\lambda}\right\rangle_{\lambda \in \Lambda^{*}}$ has a weak cluster point $x_{0} \in K$; so, by (M2), we have $x_{0} \in A$. Thus, $A \in K^{-}$. Conversely, suppose (M2) fails. Then there exists a cofinal subset $\Lambda^{\#}$ and a norm bounded net $\left\langle a_{\lambda}\right\rangle_{\lambda \in \Lambda^{*}}$ that has a weak cluster point $x_{0} \in A^{C}$ such that for each $\lambda \in \Lambda^{\#}$, we have $a_{\lambda} \in A_{\lambda}$. Choose $y \in X^{*}$ and $\alpha \in R$ such that $y\left(x_{0}\right)<\alpha<\inf \{y(a): a \in A\}$. Choose $\rho>0$ such that for each $\lambda \in \Lambda^{\#}$, we have $\left\|a_{\lambda}\right\| \leq \rho$. Then by reflexivity, $K \equiv\{x \in X: y(x) \leq \alpha\}$ and $\left.\|x\| \leq \rho\right\}$ is weakly compact, and since $x_{0}$ is a weak cluster point of $\left\langle a_{\lambda}\right\rangle_{\lambda \in \Lambda^{*}}, K$ contains $a_{\lambda}$ frequently. Thus, $A_{\lambda} \in K^{-}$frequently, whereas $A \notin K^{-}$.

We note that Lemma 5.2 could have been used to establish $(a) \Rightarrow(c)$ in the proof of Theorem 5.1, but the geometric proof that we presented seems more informative.

Theorem 5.3. Let $X$ be a reflexive Banach space. Then the graph of arg min: $\left\langle\Gamma(X), \tau_{M}\right\rangle \rightrightarrows X$ is weakly closed if and only if $X$ is finite dimensional.

Proof. If $X$ is finite dimensional, then the graph is weakly closed because it is strongly closed for any reflexive space. 
Now suppose that $X$ is infinite dimensional. Let $x_{0} \in X$ be a norm one vector, and define $f \in \Gamma(X)$ by

$$
f(x)=\left\{\begin{array}{cl}
-\alpha & \text { if } x=\alpha x_{0} \text { with } 0 \leq \alpha \leq 1 \\
\infty & \text { otherwise }
\end{array}\right.
$$

Notice that $\theta \notin \arg \min f$. We produce a net $\left\langle f_{\lambda}\right\rangle$ of proper lower semicontinuous convex functions $\tau_{M}$-convergent to $f$ such that for each $\lambda, \arg \min f_{\lambda}$ is a singleton $\left\{x_{\lambda}\right\}$ and such that $\theta=\sigma\left(X, X^{*}\right)-\lim x_{\lambda}$.

Let $\mathscr{F}$ be the collection of finite subsets of $X^{*}-\{\theta\}$, partially ordered by inclusion. Equip $\Lambda \equiv \mathscr{F} \times Z^{+}$with the product order, and define a net $\left\langle x_{\lambda}\right\rangle_{\lambda \in \Lambda}$ as follows: if $\lambda=\left(\left\{y_{1}, y_{2}, \ldots, y_{n}\right\}, k\right)$, let $x_{\lambda}$ be a point of $\left\{x \in X:\left|y_{i}(x)\right|<\right.$ $1 / k$ for $i=1, \ldots, n\}$, such that $\left\{\theta, x_{0}, x_{\lambda}\right\}$ forms an affinely independent set and $\left\|x_{\lambda}\right\|>k$. Clearly, $\theta=\sigma\left(X, X^{*}\right)-\lim x_{\lambda}$ and at the same time, $\lim _{\lambda}\left\|x_{\lambda}\right\|=\infty$. We now define $f_{\lambda}$ as follows: If $x$ is a convex combination of $\left\{\theta, x_{0}, x_{\lambda}\right\}$, say $x=\alpha_{1} \theta+\alpha_{2} x_{0}+\alpha_{3} x_{\lambda}$, then $f_{\lambda}(x)=\alpha_{2}(-1+1 / k)-\alpha_{3}$. Otherwise, we set $f_{\lambda}(x)=\infty$. Notice that $x=x_{\lambda}$ is the unique minimizer of $f_{\lambda}$, and $f_{\lambda}\left(x_{\lambda}\right)=-1$.

To show that $f=\tau_{M}-\lim f_{\lambda}$, we show that the conditions (M1) and (M2) of Lemma 5.2 are both satisfied with respect to epigraphs. Since the graph of $f$ consists of $\operatorname{co}\left(\left\{(\theta, 0),\left(x_{0},-1\right)\right\}\right)$ and the graph of $f_{\lambda}$ contains $\operatorname{co}\left(\left\{(\theta, 0),\left(x_{0},-1+1 / k\right)\right\}\right)$, where $k$ is the second coordinate of $\lambda$, it is clear that (M1) is satisfied. For (M2), suppose $\Lambda^{\#}$ is a cofinal subset of $\Lambda,\left\langle\left(w_{\lambda}, \beta_{\lambda}\right)\right\rangle_{\lambda \in \Lambda^{*}}$ is a uniformly bounded net such that for each $\lambda \in \Lambda^{\#}$, $\left(w_{\lambda}, \beta_{\lambda}\right) \in \operatorname{epi} f_{\lambda}$, and $\left\langle\left(w_{\lambda}, \beta_{\lambda}\right)\right\rangle_{\lambda \in \Lambda^{*}}$ has $(w, \beta)$ as a weak cluster point. We may write

$$
w_{\lambda}=\mu_{\lambda} x_{\lambda}+\left(1-\mu_{\lambda}\right)\left(\alpha_{\lambda} x_{0}\right),
$$

where $\mu_{\lambda} \in[0,1]$ and $\alpha_{\lambda} \in[0,1]$. By passing to a subnet, we may assume that $\left\langle\left(w_{\lambda}, \beta_{\lambda}\right)\right\rangle_{\lambda \in \Lambda^{*}}$ converges weakly to $(w, \beta)$, that $\left\langle\mu_{\lambda}\right\rangle_{\lambda \in \Lambda^{*}} \rightarrow \mu_{0}$, and that $\left\langle\alpha_{\lambda}\right\rangle_{\lambda \in \Lambda^{*}} \rightarrow \alpha_{0}$. Since $\left\langle w_{\lambda}\right\rangle_{\lambda \in \Lambda^{*}}$ and $\left\langle\alpha_{\lambda}\right\rangle_{\lambda \in \Lambda^{*}}$ are uniformly bounded and $\lim _{\lambda \in \Lambda^{*}}\left\|x_{\lambda}\right\| \rightarrow \infty$, we conclude that $\mu_{0}=0$. Since addition and scalar multiplication are jointly continuous, we have

$$
w=\sigma\left(X, X^{*}\right)-\lim w_{\lambda}=0 \cdot \theta+1 \cdot \alpha_{0} x_{0}=\alpha_{0} x_{0} .
$$

Since $\beta_{\lambda} \geq f_{\lambda}\left(w_{\lambda}\right)$ for each $\lambda \in \Lambda^{\#}$, it follows that

$$
\begin{aligned}
\beta=\lim _{\lambda \in \Lambda^{*}} \beta_{\lambda} & \geq \lim \sup _{\lambda \in \Lambda^{*}} f_{\lambda}\left(\mu_{\lambda} x_{\lambda}+\left(1-\mu_{\lambda}\right)\left(\alpha_{\lambda} x_{0}\right)\right) \\
& =\lim \sup _{\lambda \in \Lambda^{*}} \mu_{\lambda} f_{\lambda}\left(x_{\lambda}\right)+\alpha_{\lambda}\left(1-\mu_{\lambda}\right)\left(f_{\lambda}\left(x_{0}\right)\right) \\
& =0(-1)+\alpha_{0} \cdot 1 \cdot(-1)=-\alpha_{0}=f(w) .
\end{aligned}
$$

This proves that $(w, \beta) \in \operatorname{epi} f$. Thus, (M2) is verified and $f=\tau_{M}-\lim f_{\lambda}$. Since $\left(f_{\lambda}, x_{\lambda}\right)$ is in the graph of arg min for each $\lambda$ and $(f, \theta)$ is not in the graph of arg min, the multifunction fails to have weakly closed graph. 
In [Be3], a net in $\left\langle A_{\lambda}\right\rangle$ in $\mathscr{C}(X)$ was called topologically Mosco convergent to $A \in \mathscr{C}(X)$ provided both of the following conditions are met: (a) for each $a \in A$, each strong (norm) neighborhood of $a$ meets $\left\langle A_{\lambda}\right\rangle$ eventually, and (b) if each weak neighborhood of a point $x \in X$ meets $\left\langle A_{\lambda}\right\rangle$ frequently, then $x \in A$. This parallels the usual definition of topological convergence of a net of sets in a topological space (see [KT, p. 24]). In view of Lemma 5.2, the conjunction of (a) and (b) is formally stronger than $A=\tau_{M}-\lim A_{\lambda}$. The last example shows that (a) and (b) together are in fact stronger, because each weak neighborhood of $(\theta,-1)$ meets $\left\langle\right.$ epi $f_{\lambda}$ 〉 eventually, whereas $(\theta,-1) \notin$ epi $f$.

The thrust of this article is that Mosco convergence is probably not worthy of study outside the reflexive setting. An alternative to $\tau_{M}$ for general Banach spaces is the topology of uniform convergence of distance functions on bounded subsets of $X$, which is a stronger completely metrizable topology on $\mathscr{C}(X)$ for any Banach space $X$ [ALW], and which is stable with respect to duality, without reflexivity, or even completeness [Be4].

\section{REFERENCES}

[At] H. Attouch, Variational convergence for functions and operators, Pitman Publishers, Boston, 1984.

[ALW] H. Attouch, R. Lucchetti, and R. Wets, The topology of the $\rho$-Hausdorff distance, Annali Mat. Pura Appl. (to appear).

[BP] M. Baronti and P.-L. Papini, Convergence of sequences of sets, in Methods of functional analysis in approximation theory, ISNM 76, Birkhäuser Verlag, 1986.

[Be1] G. Beer, On Mosco convergence of convex sets, Bull. Australian Math. Soc. 38 (1988), 239253.

[Be2] - On the Young-Fenchel transform for convex functions, Proc. Amer. Math. Soc. 104 (1988), 1115-1123.

[Be3] - Support and distance functionals for convex sets, Numer. Funct. Anal. Opt. 10 (1989), 15-36.

[Be4] _ Conjugate convex functions and the epi-distance topology, Proc. Amer. Math. Soc. (to appear).

[BF] J. Borwein and S. Fitzpatrick, Mosco convergence and the Kadec property, Proc. Amer. Math. Soc. 106 (1989), 843-852.

[Da] M. M. Day, Normed linear spaces, 3rd ed., Springer-Verlag, New York, 1973.

[Ho] R. Holmes, A course in optimization and best approximation, Lecture Notes in Math., vol. 257, Springer-Verlag, New York, 1972.

[Ja] R. C. James, Reflexivity and the supremum of linear functionals, Israel J. Math. 13 (1972), 289-300.

[KT] E. Klein and A. Thompson, Theory of correspondences, Wiley, Toronto, 1984.

[LP] R. Lucchetti and F. Patrone, Hadamard and Tyhonov well-posedness of a certain class of convex functions, J. Math. Anal. Appl. 88 (1982), 204-215.

[Mc] L. McLinden, Successive approximation and linear stability involving convergent sequences of optimization problems, J. Approximation Theory 35 (1982), 311-354.

[Mo1] U. Mosco, Convergence of convex sets and solutions of variational inequalities, Adv. in Math. 3 (1969), 510-585.

[Mo2] _ On the continuity of the Young-Fenchel transform, J. Math. Anal. Appl. 35 (1971), 518-535. 
[SW] G. Salinetti and R. Wets, On the relation between two types of convergence for convex functions, J. Math. Anal. Appl. 60 (1977), 211-226.

[So] Y. Sonntag, Convergence au sens de Mosco, théorie et applications à l'approximation des solutions d'inéquations, Thèse d'Etat, Université de Provence, Marseille, 1982.

[Ts] M. Tsukada, Convergence of best approximations in a smooth Banach space, J. Approximation Theory 40 (1984), 301-309.

[Wi] R. Wijsman, Convergence of sequences of convex sets, cones, and functions II, Trans. Amer. Math. Soc. 123 (1966), 32-45.

Department of Mathematics, California State University, Los Angeles, Los Angeles, CALIFORNia 90032

Department of Mathematics, Statistics and Computing Science, Dalhousie UniverSity, Halifax, Nova Scotia B3H 3J5 Canada 